\title{
COMPUTING THE TOPOLOGICAL ENTROPY OF GENERAL ONE-DIMENSIONAL MAPS
}

\author{
P. GÓRA AND A. BOYARSKY
}

\begin{abstract}
A matrix-theoretic method for computing the topological entropy of continuous, piecewise monotonic maps of the interval is presented. The method results in a constructive procedure which is easily implemented on the computer. Examples for families of unimodal, nonunimodal and discontinuous maps are presented.
\end{abstract}

\section{INTRODUCTION}

The measure-theoretic entropy (metric entropy), the Lyapunov exponent and the topological entropy are some of the quantitators of irregular behavior in dynamical systems. Metric entropy is a classical tool of information theory. The orbits of a map with low metric entropy tends to be predictable with some degree of accuracy, while this is not the case for maps with high metric entropy. The Lyapunov exponent is defined to be the eigenvalue of the derivative of the map $f$ averaged along orbits. A large derivative reflects large expansion, implying that nearby points are quickly pushed apart. Hence the Lyapunov exponent reflects dependence on initial conditions. That topological entropy is a measure of chaotic behavior is evidenced by the fact that if $h(f)>0$, then a phenomenon similar to the horseshoe must exist [3]. Interrelations between metric entropy and the Lyapunov exponent are given in [12].

While estimating metric entropy and the Lyapunov exponent has been the subject of intense research in the physics literature (see the references in [8]), the computation of topological entropy has only recently received attention. Using the kneading theory of [4], an algorithm for computing $h(f)$ was presented in [1], where $f$ is continuous and unimodal. An improved algorithm using kneading theory was given in [2]. The methods of both [1] and [2] depend critically on a certain ordering lemma (Lemma 2 of [1]), which is known to be true only for continuous, unimodal maps. In [13], topological entropy is computed using the periodic points of the transformation.

For a piecewise linear Markov map, $f$, the topological entropy is the maximal eigenvalue of the induced $0-1$ matrix $M_{f}$. The method of this note uses

Received by the editors February 1, 1989.

1980 Mathematics Subject Classification (1985 Revision). Primary 28A65; Secondary 58F11.

The research of the second author was supported by NSERC and FCAR grants. 
this fact to compute the topological entropy of general piecewise monotonic maps. Let $f$ be any piecewise monotonic map and let $\left\{g_{n}\right\}$ be a sequence of piecewise monotonic maps each of which has the same number of monotonic pieces as $f$. The main result shows that $\lim _{n \rightarrow \infty} h\left(g_{n}\right)=h(f)$. Thus, $h(f)$ is computed by finding the maximal eigenvalues of the matrices $M_{g_{n}}$. The main advantage of this method is the use of preimages of turning points to define the matrix $M_{g_{n}}$. (In [13] the constructed approximating matrices use periodic points, which are much harder to find.)

This idea was used in $[8,9]$ to estimate the absolutely continuous invariant measures of piecewise monotonic expanding and piecewise monotonic, nonexpanding maps, as well as the corresponding Lyapunov exponents. In this context it is interesting to observe that $M_{f}$ codes for two types of information

-the Lyapunov exponent through the absolutely continuous invariant measure

-and the topological entropy.

Consider, for example, the skewed triangle map on $[0,1]$ and let $f_{0}$ denote the symmetric triangle map. In [10], it is shown that $f$ is topologically conjugate to $f_{0}$ via a homeomorphism which is not absolutely continuous. Hence $h(f)=$ $\log _{2} 2$ and the absolutely continuous invariant measure of $f$, Lebesgue measure, is not maximal. Since $f$ is topologically conjugate to $f_{0}, M_{f}$ is the same as $M_{f_{0}}$, i.e.,

$$
M_{f}=\left(\begin{array}{ll}
1 & 1 \\
1 & 1
\end{array}\right) .
$$

The Frobenius-Perron operator $P_{f}$ is then directly computed from $M_{f}$ and the slopes of $f$, and the fixed point of $P_{f}$ is the density of the absolutely continuous invariant measure. We observe that since the maximal measure is singular with respect to Lebesgue measure, the topological entropy is not visible to physical observation, yet it is given by the matrix $M_{f}$, which yields the observable dynamics associated with the absolutely continuous invariant measure [12].

We present our main result in $\S \S 2$ and 3. We construct an invariant set $X$ which is a subset of the closure of the set of all preimages of turning points of $f$, and we prove that for a sequence $\left\{g_{k}\right\}$ of maps approximating $f$ on $X$, under some additional conditions (see Theorem 2 and Corollary 1), we have

$$
h(f)=\lim _{k \rightarrow \infty} h\left(g_{k}\right) .
$$

In $\S 3$, an algorithm based on the main result is presented. Examples of unimodal, nonunimodal and discontinuous maps are discussed in $\S 4$.

\section{MAIN RESUlt}

Let $I=[0,1]$ and let $X$ be a closed subset of $I$. A map $f: X \rightarrow X$ is called a p.m. map if it is continuous and piecewise monotonic, i.e., if there exist 
points $0=x_{0}<x_{1}<\cdots<x_{c_{1}}=1$ in $X$ such that

$$
f_{\left[\left[x_{i-1}, x_{i}\right] \cap X\right.} \text { is monotonic, } i=1, \ldots, c_{1}+1 \text {. }
$$

The points $x_{0}, \ldots, x_{c_{1}}$ are called turning points of $f$. The endpoints of $I$ are considered as turning points. For a p.m. map $f$, we define $c_{m}=c\left(f^{m}\right)$ to be the number of intervals of monotonicity of $f^{m}=f \circ f \circ \cdots \circ f$, the $m$ th iterate of $f$. It is easy to see that $f^{m}$ is a p.m. map if $f$ is.

In the sequel, we shall need the following results of Misiurewicz and Szlenk [3] :

Theorem A. If $f: X \rightarrow X$ is a p.m. transformation, then

$$
h(f)=\lim _{k \rightarrow \infty} \frac{1}{k} \log _{2} c\left(f^{k}\right)=\inf _{k} \frac{1}{k} \log _{2} c\left(f^{k}\right) .
$$

Let $\mathscr{C}^{0}(X, X)$ denote the space of continuous functions from $X$ into $X$ with the standard norm. Denote by $\mathscr{J}$ the set of all subintervals of $I$. For $X \subset I, \mathscr{J}_{\mid X}$ denotes the family of sets $\{J \cap X: J \in \mathscr{J}\}$. A map $f \in \mathscr{C}^{0}(X, X)$ has the Darboux property if for any $J \in \mathscr{J}_{\mid X}$ we have $f(J) \in \mathscr{J}_{\mid X}$.

The following Theorem B is a rephrased version of Theorem 5 of Misiurewicz and Szlenk [3] .

Theorem B. Let $f: I \rightarrow I$ be a continuous mapping and let $X$ be a closed invariant subset of I such that $h\left(f_{\mid X}\right)=h(f)$ and $f_{\mid X}: X \rightarrow X$ is p.m. and has the Darboux property. Then for any sequence $\left\{g_{n}\right\} \subset \mathscr{C}^{0}(I, I)$, such that $g_{n} \rightarrow f$ in $\mathscr{C}^{0}(X, X)$ we have

$$
h(f) \leq \liminf _{n} h\left(g_{n}\right) .
$$

If $f \in \mathscr{C}^{0}(I, I)$ and $X$ is both forward and backward invariant under $f$, then $f_{\mid X}$ has the Darboux property. To prove this, let $J \in \mathscr{J}$ and $A=J \cap X$. Since $X$ is forward invariant we have $f(A) \subset f(J) \cap X$. Since $X$ is backward invariant we have

$$
f^{-1}(f(J) \cap X) \cap J \subset X \cap J=A,
$$

so $f(J) \cap X \subset f(A)$. Thus $f(J) \cap X=f(A)$.

An interval $J$ is called a homterval if, for any positive integer $n, f_{\mid J}^{n}$ is a homeomorphism onto its image. It is obvious that the image of a homterval is a homterval. For a p.m. $f$, the preimage of a homterval is either a homterval or a union of a finite number of homtervals together with some turning points of $f$.

Let $H(f)$ be the family of all open maximal homtervals of $f$. It is easy to see that if $J_{1}, J_{2} \in H(f)$ and $f\left(J_{1}\right) \cap J_{2} \neq \varnothing$ then $f\left(J_{1}\right) \subset J_{2}$.

We divide $H(f)$ into two subfamilies:

$$
H_{W}(f)=\left\{J \in H(f): f^{k}(J) \cap J=\varnothing, \text { for any } k\right\}
$$

and 


$$
H_{R}(f)=\left\{J \in H(f): f^{k}(J) \subseteq J \text {, for some } k\right\} .
$$

If $J \in H_{R}(f)$ we define $k(J)$ as the smallest $k$ such that $f^{k}(J) \subseteq J$.

Lemma 1. Let

$$
Y_{1}=\left\{\bigcup_{J} \bigcup_{k=0}^{\infty} f^{-k}(J): J \in H_{W}(f)\right\}
$$

and

$$
\begin{aligned}
& Y_{2}=\left\{\bigcup_{J} \bigcup_{j=1}^{\infty} f^{-j}(J): J \in H_{R}(f), f^{-j}(J) \cap f^{k}(J)=\varnothing\right. \\
& \qquad \begin{aligned}
\text { for } k & =0,1, \ldots, k(J)-1\} .
\end{aligned}
\end{aligned}
$$

Then the set $Y=Y_{1} \cup Y_{2}$ is an open subset of the set of wandering points $W(f)$. Moreover, $f(I \backslash Y) \subset I \backslash Y$ and $h\left(f_{\mid I \backslash Y}\right)=h(f)$.

Proof. If $x \in J \in H_{W}(f)$, then $x$ is a wandering point, since $J$ is a neighbourhood of $x$ and no image $f^{k}(J)$ intersects $J$. If $x \in Y_{2}$, a neighbourhood of $x$ having the form $f^{-j}(J)$, where $J \in H_{R}(f)$, is mapped by $f^{j}$ into $J$ and stays forever in the set $\bigcup_{k=0}^{k(J)-1} f^{k}(J)$, which is disjoint from $f^{-j}(J)$. Thus $x$ is a wandering point. Since any preimage of a wandering point is a wandering point itself, $Y \subset W(f)$. The fact that $h\left(f_{\mid I \backslash W(f)}\right)=h(f)$ is well known [14, Corollary 8.6.1.]. The rest of the proof is straightforward.

We define

$$
X_{1}=\mathrm{cl}\left(\bigcup_{k=0}^{\infty} f^{-k}\left\{x_{0}, \ldots, x_{c_{1}}\right\}\right),
$$

where $x_{0}, \ldots, x_{c_{1}}$ are the turning points of $f$.

Lemma 2. $I \backslash X_{1}=\{\bigcup J: J \in H(f)\}$.

Proof. $J \in H(f)$ if and only if it includes no preimages of turning points of $f$.

Let $A$ denote the set of isolated points of $X_{1}$. Any point in $A$ is a preimage of a turning point of $f$ or a turning point itself. We divide $A$ into two subsets:

$$
A_{1}=\left\{x \in A: f^{k}(x) \in J, \text { for some } k \text { and some } J \in H(f)\right\}
$$

and $A_{2}=A \backslash A_{1}$. Obviously $A_{1} \subset Y$, so these points are unimportant.

Lemma 3. Let $x_{i}$ be a turning point of $f$ belonging to $A_{2}$. Then either $f\left(x_{i}\right)=$ $f^{-k}\left(x_{j}\right)$ for a turning point $x_{j}$ of $f$ and a positive integer $k$ or $f\left(x_{i}\right) \in X_{1} \backslash A$. Proof. Since $x_{i} \in A_{2}, f\left(x_{i}\right)$ is an endpoint of a maximal homterval.

Now we are ready to define the set: $X=X_{1} \backslash A_{1}$. 
Theorem 1. The set $X$ is closed, forward and backward invariant, and $h\left(f_{\mid X}\right)=$ $h(f)$.

Proof. We have

$$
I=X \cup Y \cup\left\{\bigcup J: J \in H_{R}(f)\right\} .
$$

$X$ is closed by definition. It is forward and backward invariant since its complement has these properties. By Lemma 1 we have $h(f)=h\left(f_{\mid I \backslash Y}\right)$. Since for any $J \in H_{R}(f), f_{\mid J}^{k(J)}$ is a homeomorphism, we have $h\left(f_{\mid \bar{J}}^{k(J)}\right)=0 . \quad I \backslash Y$ is a union of disjoint forward invariant sets

$$
I \backslash Y=X \cup \bigcup_{n=1}^{\infty} J_{n}, \quad J_{n} \in H_{R}(f) .
$$

Hence $h\left(f_{\mid I \backslash Y}\right)=h\left(f_{\mid X}\right)$.

The following theorem is proved in [3].

Theorem C. Let $f$ be a $\mathscr{C}^{2}$ p.m. mapping such that for any $x \in I$ at least one of the numbers $f^{\prime}(x), f^{\prime \prime}(x)$ is nonzero. Then the topological entropy regarded as a function $h: \mathscr{C}^{2}(I, I) \rightarrow \overline{\mathbb{R}}$ is upper semicontinuous at $f$, i.e.:

$$
h(f) \geq \limsup _{n} h\left(g_{n}\right),
$$

for any sequence $\left\{g_{n}\right\} \subset \mathscr{C}^{2}(I, I)$, such that $g_{n} \rightarrow f$ in $\mathscr{C}^{2}(I, I)$.

We now prove an analogue of Theorem $C$ by weakening the smoothness assumption at the price of some additional conditions. Let $f: X \rightarrow X$ be a p.m. mapping. Let $\mathscr{C}(f)$ denote the set of p.m. mappings which have the same number of turning points as $f$.

Theorem 2. Let $f: X \rightarrow X$ be a p.m. continuous mapping such that no turning point of $f$ is periodic. Then for any sequence $\left\{g_{n}\right\} \subset \mathscr{C}(f)$, such that $g_{n} \rightarrow f$ in $\mathscr{C}^{0}(X, X)$ we have

$$
h(f) \geq \limsup _{n} h\left(g_{n}\right) .
$$

We will need the following two lemmas:

Lemma 4. Let $f: X \rightarrow X$ be a p.m. mapping. If $x$ is a turning point of $f$, then for any small enough $\eta>0$, we can find a $\mathscr{C}^{0}$-neighbourhood $\mathscr{U}$ of $f$, such that any $g \in \mathscr{U}$ has a turning point in $(x-\eta, x+\eta) \cap X$.

Proof. Assume $f$ has a local minimum at $x$. Then there exists an $\eta>0$ such that $f$ is decreasing on $(x-\eta, x) \cap X$, and $f$ is increasing on $(x, x+\eta) \cap X$. Let $x^{-}$be a point in $(x-\eta, x) \cap X$ such that $f\left(x^{-}\right)>f(x)$, and let $x^{+}$be a point in $(x, x+\eta) \cap X$ such that $f\left(x^{+}\right)>f(x)$. Let

$$
\varepsilon=\frac{1}{3} \min \left\{f\left(x^{-}\right)-f(x), f\left(x^{+}\right)-f(x)\right\} .
$$

If $g \in \mathscr{C}^{0}(X, X)$ and $\|g-f\|_{\ell^{0}}<\varepsilon$, then

$$
g(x) \leq f(x)+\varepsilon, \quad g\left(x^{-}\right) \geq f\left(x^{-}\right)-\varepsilon,
$$


and

$$
g\left(x^{+}\right) \geq f\left(x^{+}\right)-\varepsilon .
$$

Thus $g(x)<\min \left\{g\left(x^{-}\right), g\left(x^{+}\right)\right\}$and $g$ has a local minimum in $(x-\eta, x+$ $\eta) \cap X$.

Lemma 5. Let $f: X \rightarrow X$ be a p.m. transformation. Let $x \in I$ be the point such that $f$ is monotonic on $(x-\delta, x+\delta) \cap X, \delta>0$. Then for $g \in \mathscr{C}(f)$ and close enough to $f$ in $\mathscr{C}^{0}, g$ is monotonic on $\left(x-\delta_{1}, x+\delta_{1}\right) \cap X$ for any $\delta_{1}<\delta$.

Proof. Let $\delta_{1}<\delta$ be fixed and let $\gamma$ be the distance from $x$ to the nearest turning point of $f$, say $x_{i}$. Let $\eta=\gamma-\delta_{1}$. For this $\eta$, we can find a neighbourhood $\mathscr{U}$ of $f$ in $\mathscr{C}^{0}$ such that for any $g \in \mathscr{U}$ has a turning point in $\left(x_{i}-\eta, x_{i}+\eta\right) \cap X$. Since $g \in \mathscr{C}(f)$, it has the same number of turning points as $f$. Therefore, $g$ is monotonic on $\left(x-\delta_{1}, x+\delta_{1}\right) \cap X$.

Proof of Theorem 2. Let us fix $\varepsilon>0$. Since $h(f)=\inf _{m}\left\{\frac{1}{m} \log c_{m}\right\}$, there exists a positive integer $m$ such that

$$
\frac{1}{m} \log c_{m} \leq h(f)+\varepsilon / 2 .
$$

Let $x_{1}, \ldots, x_{c_{m}}$ be all the turning points of $f^{m}$. Let $g \in \mathscr{C}(f)$ be close to $f$ in $\mathscr{C}^{0}(I, I)$. Let $x$ be a turning point of $g^{m}$. We have

$$
g^{m}(x+t)=g(g(\cdots g(x+t) \cdots)) .
$$

for $t$ in $\left(-\delta_{1}, \delta_{2}\right), \delta_{1}, \delta_{2}>0,(x+t) \in X$. Since the composition of monotonic maps is a monotonic map, at least one of the points $g^{j}(x), j=$ $0,1, \ldots, m-1$, is a turning point for $g$. Suppose it is $g^{k}(x)$. By Lemma $5, g^{k}(x)$ is close to a turning point of $f$, say $\bar{x}$. Now, $y=f^{-k+1}(\bar{x})$ is a turning point of $f^{m}$. Since $m$ is fixed and $g$ is close to $f$, there exists a $y$ which is close to $x$ and the trajectories $\left\{x, g(x), \ldots, g^{m-1}(x)\right\}$ and $\left\{y, f(y), \ldots, f^{m-1}(y)\right\}$ are close. So the trajectory $\left\{x, g(x), \ldots, g^{m-1}(x)\right\}$ lies close to the trajectory of one of the turning points of $f^{m}$.

Let $r_{i}$ denote the number of turning points of $f$ in

$$
\left\{x_{i}, f\left(x_{i}\right), \ldots, f^{m-1}\left(x_{i}\right)\right\}, \quad i=1, \ldots, c_{m} .
$$

If $g$ is close enough to $f, g^{m}$ can have at most $2^{r_{i}}$ turning points $x$, whose orbits are close to the orbit of $x_{i}$-one for each pattern of turning points in $\left\{x, g(x), \ldots, g^{m-1}(x)\right\}$. Since $f$ has no turning periodic points, we have $r_{i} \leq c_{1}, \quad i=1, \ldots, c_{m}$, and $c\left(g^{m}\right) \leq c\left(f^{m}\right) \cdot 2^{c_{1}}$. Thus we have

$$
\begin{aligned}
h(g) & \leq \frac{1}{m} \log c\left(g^{m}\right) \leq \frac{1}{m} \log c\left(f^{m}\right)+\frac{1}{m} \log 2^{c_{1}} \\
& \leq h(f)+\frac{\varepsilon}{2}+\frac{1}{m} \log 2^{c_{1}} .
\end{aligned}
$$


Since $\varepsilon$ is arbitrary and we can choose $m$ as big as we wish, the proof of the theorem is complete.

Corollary 1. Let $f: X \rightarrow X$ be a p.m. continuous mapping. Let $\mathscr{C}_{p}(f)=\{g \in$ $\mathscr{C}(f)$ : any periodic turning point of $f$ is a turning point of $g$ and $g(x)=f(x)$ for any point in the trajectory of a turning periodic point of $f\}$. Then for any sequence $\left\{g_{n}\right\} \subset \mathscr{C}_{p}(f)$, such that $g_{n} \rightarrow f$ in $\mathscr{C}^{0}(X, X)$ we have

$$
h(f) \geq \limsup _{n} h\left(g_{n}\right) \text {. }
$$

Proof. Follows directly from the proof of Theorem 2.

\section{The Algorithm}

We now describe the algorithm which is based on the theoretical results of $\S 2$. Let $f: I \rightarrow I$ be a continuous piecewise monotonic map. Let $\mathscr{I}=\left\{I_{i}\right\}_{i=1}^{n}$ be the intervals of monotonicity and let $Q$ denote the turning points of $f$.

Step 1. Set $\mathscr{I}^{(k)}=\bigvee_{i=0}^{k} f^{-i}(\mathscr{I})$, and let $\left\{I_{i}^{(k)}\right\}$ denote the intervals in $\mathscr{I}^{(k)}$. Let $Q^{(k)}$ be the set of endpoints of the intervals in $\mathscr{I}^{(k)}$. Suppose there are $n_{k}+1$ points in $Q^{(k)}$, then there are $n_{k}$ intervals in $\mathscr{I}^{(k)}$.

Step 2. Form the map $g_{k}$ on the partition of $\mathscr{I}^{(k)}$ as follows: if $a \in \mathscr{Q}^{(k)} \backslash \mathscr{Q}$. Let $g_{k}(a)=f(a)$; if $a \in \mathscr{Q}$, let $g_{k}(a)$ be the point in $\mathscr{Q}^{(k)}$ which is closest to $f(a)$. Connect all the adjoining points $\left\{\left(a, g_{k}(a)\right): a \in \mathscr{Q}^{(k)}\right\}$ by straight lines. This defines a piecewise linear map $g_{k}$, which is Markov and which approximates $f$.

Step 3. Form the square matrix $M_{k}$ as follows: the $i j$ th entry of $M_{k}$ is 1 if $g_{k}\left(I_{i}^{(k)}\right) \supset I_{j}^{(k)}$ and is 0 otherwise.

Step 4. Compute the maximal eigenvalue of $M_{k}$. This is done by the following standard procedure. Let $v=(1,1, \ldots, 1)$. Compute $v_{1}=v M_{k}$ and $\left\|v_{1}\right\|$, the Euclidean norm of $v_{1}$. Define $v_{i+1}=\left(v_{i} /\left\|v_{i}\right\|\right) M_{k}, i=1,2, \ldots$ Then $\left\|v_{i}\right\| \rightarrow \lambda_{k}$, the maximal eigenvalue of $M_{k}$, as $i \rightarrow \infty$.

Theorem 3. Let $f: I \rightarrow I$ be a p.m. continuous map and $\left\{g_{k}\right\}_{k=1}^{\infty}$ a sequence of piecewise linear Markov map constructed by the algorithm. Then

$$
h(f)=\lim _{k \rightarrow \infty} h\left(g_{k}\right) \text {. }
$$

Proof. First we will prove that $g_{k} \rightarrow f$ in $\mathscr{C}^{0}(X, X)$, where $X$ is the set defined in $\S 2$. Since $g_{k}=f$ on $Q^{(k)} \backslash Q, g_{k} \rightarrow f$ on $X \backslash(Q \cap X)$. We have to prove that $g_{k}\left(x_{i}\right) \rightarrow f\left(x_{i}\right)$ for any turning point $x_{i}$ of $f$ which belongs to $A_{2}$. By Lemma $3, f\left(x_{i}\right)$ is either a preimage of a turning point of $f$, and then $g_{k}\left(x_{i}\right)=f\left(x_{i}\right)$ for $k$ big enough, or $f\left(x_{i}\right) \in X_{1} \backslash A$ and then this value is approximated by $g_{k}\left(x_{i}\right)$ by the construction used in the algorithm. Let us also notice that for $k$ big enough $g_{k} \in \mathscr{C}_{p}(f)$. We proved that we can invoke Theorem B and Corollary 1 . Theorem 3 is thus proved. 
Remark 1. Let us assume that for every turning point $x_{i} \in Q$ which belongs to $A_{2}$, we have $f\left(x_{i}\right) \notin X_{1} \backslash A$. This occurs, for example, if $A_{2}$ is empty. (This will be guaranteed if there are no homtervals.) In this case, we can choose the approximating piecewise linear Markov maps $\left\{g_{k}\right\}$ in such a way that $g_{k}\left(x_{i}\right) \leq$ $f\left(x_{i}\right)$ if $f$ has a local maximum at $x_{i}$, and $g_{k}\left(x_{i}\right) \geq f\left(x_{i}\right)$ if $f$ has a local minimum at $x_{i}$. Then

$$
h\left(g_{k}\right) \leq h(f), \quad k=1,2, \ldots,
$$

as is proved in [6]. Since

$$
\frac{1}{k} \log c\left(f^{k}\right) \geq h(f), \quad k=1,2, \ldots,
$$

we obtain a two-sided estimate of $h(f)$.

Remark 2. This algorithm was implemented on a PC AT using matrices up to dimension 256. A number of examples are presented in $\S 5$.

\section{ENTROPY OF DISCONTINUOUS MAPS}

Let $f: I \rightarrow I$ be a piecewise monotonic map, not necessarily continuous. Let $g_{n}$ be a piecewise linear map with the same number of monotonic pieces as $f$, i.e., $g_{n} \in \mathscr{C}(f)$.

Definition. We say that $f: I \rightarrow I$ has topological entropy $h(f)$ if there exists a sequence of piecewise linear Markov maps $\left\{g_{n}\right\} \subset \mathscr{C}(f),\left\|g_{n}-f\right\|_{\mathscr{E}^{0}} \rightarrow 0$, such that $\left\{\log \lambda_{n}\right\}$ converges to $h(f)$, where $\lambda_{n}$ are the maximal eigenvalues of the $0-1$ matrices induced by $\left\{g_{n}\right\}$.

If $f$ is continuous, we have already shown that this is the case. To see that $h(f)$ is well defined, let $\left\{g_{n}^{1}\right\}$ and $\left\{g_{n}^{2}\right\}$ be two different sequences in $\mathscr{C}(f)$ such that $\left\|g_{n}^{1}-f\right\|_{\digamma^{0}} \rightarrow 0,\left\|g_{n}^{2}-f\right\|_{\mathscr{F}^{0}} \rightarrow 0$ as $n \rightarrow \infty$, but $\lambda_{n} \rightarrow \lambda$ and $\beta_{n} \rightarrow \beta$, where $\lambda \neq \beta$, and $\lambda_{n}, \beta_{n}$ are the maximal eigenvalues of the $0-1$ matrices induced by $\left\{g_{n}^{1}\right\}$ and $\left\{g_{n}^{2}\right\}$, respectively. Thus, there exists an $\varepsilon>0$ and an integer $N$ such that $\left|\lambda_{n}-\beta_{n}\right|>\varepsilon$ for all $n>N$. But this implies that the associated 0-1 matrices and the maps which induce them are separated, i.e., $\left\|g_{n}^{1}-g_{n}^{2}\right\|_{\digamma^{0}}>a>0$ as $n \rightarrow \infty$, which is false. Hence $h(f)$ is well-defined.

Proposition 1. Let $f$ be as above and let $h(f)$ be its topological entropy. Then $h(f)$ is invariant under topological conjugation.

Proof. Let $h: I \rightarrow I$ be a homeomorphism and let $T=h \circ f \circ h^{-1}$. Let $\left\{g_{n}\right\}$ be a sequence of piecewise linear Markov maps in $\mathscr{C}(f)$ which converges uniformly for $f$. Define $\bar{T}_{n}=h \circ g_{n} \circ h^{-1}$. Then $\bar{T}_{n}$ is Markov and is in $\mathscr{C}(T)$, but it is not piecewise linear. Furthermore, $\left\|\bar{T}_{n}-T\right\|_{\mathscr{F}^{0}} \rightarrow 0$. 
Now since $\bar{T}_{n}$ is topologically conjugate to $g_{n}$, the maximal eigenvalues of the 0-1 matrices induced by $\bar{T}_{n}$ and $g_{n}$ are the same, say $\lambda_{n}$. Fix $n$ for the the moment and let $\left\{T_{n, j}\right\}$ be a sequence of piecewise linear Markov maps in $\mathscr{C}\left(\bar{T}_{n}\right)$ and such that $\left\|T_{n, j}-T_{n}\right\|_{\mathscr{\digamma}^{0}} \rightarrow 0$ as $j \rightarrow \infty$. Then $\lambda_{n, j} \rightarrow \lambda_{n}$ as $j \rightarrow \infty$. Define $T_{n}=T_{n, n}$. Then $\left\{T_{n}\right\}$ is a sequence of piecewise linear Markov maps in $\mathscr{C}(T)$ and $\left\|T_{n}-T\right\|_{\mathscr{F}^{0}} \rightarrow 0$ as $n \rightarrow \infty$. Let $\beta_{n}=\lambda_{n, n}$. Since $\lambda_{n} \rightarrow$ $h(f), \beta_{n} \rightarrow h(f)$.

\section{EXAMPLES}

1. Let $f_{r}(x)=r x(1-x)$ be the quadratic family of maps on $[0,1]$, with $r \in[3.58,4.00]$. For these parameter values $h\left(f_{r}\right)>0$. Figure 1 shows the plot of $h\left(f_{r}\right)$ versus $r$, which accords well with the plot in [2].

2. Let $f_{r}(x)=r(\sin 2 \pi+1), r \in[.3, .5]$, be a family of maps on $[0,1]$. Each $f_{r}$ is a bimodal function and hence the results of [2] do not apply here. Figure 2 shows the plot of $h\left(f_{r}\right)$ versus $r$.

3. Let

$$
f_{r}(x)= \begin{cases}4 x(1-x), & \text { if } 0 \leq x \leq .5 \\ r x(1-x), & \text { if } .5<x \leq 1,\end{cases}
$$

where $r \in[3.58,4.00]$. For $r<4, f_{r}(x)$ is discontinuous. Figure 3 shows the plot of $h\left(f_{r}\right)$ versus $r$ for $r \in[3.58,4.00]$. We show only points since it is not known that $h\left(f_{r}\right)$ is continuous as a function of $r$.

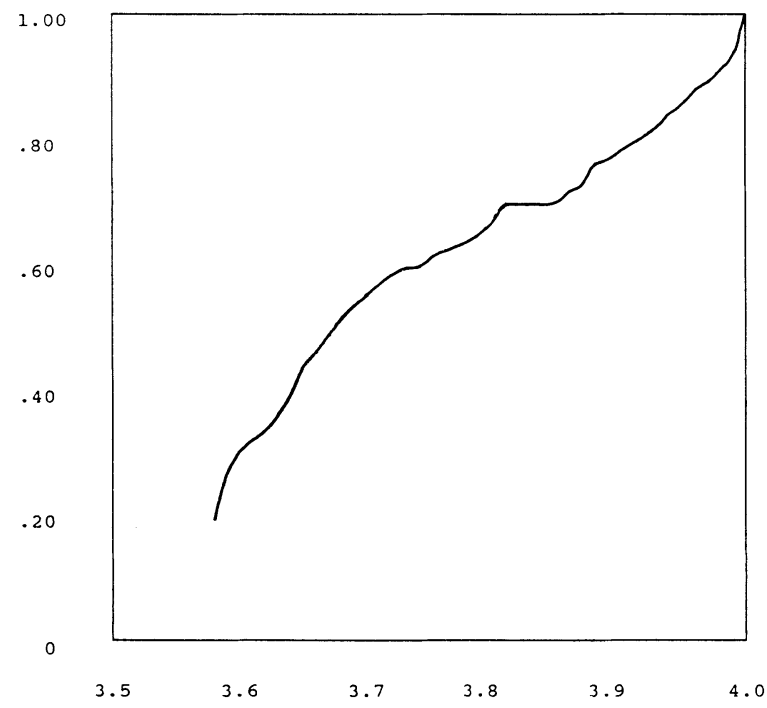

FIGURE 1

Computed topological entropy of the family $r x(1-x)$ as a function of the parameter $r$ 


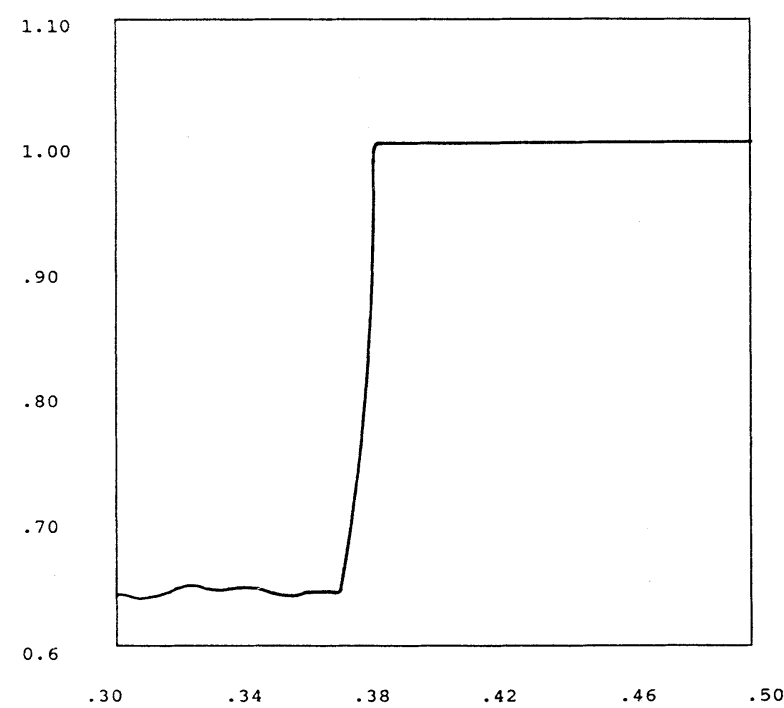

FIGURE 2

Computed topological entropy of the family $r(\sin (2 \pi x)+1)$ as a function of the parameter $r$

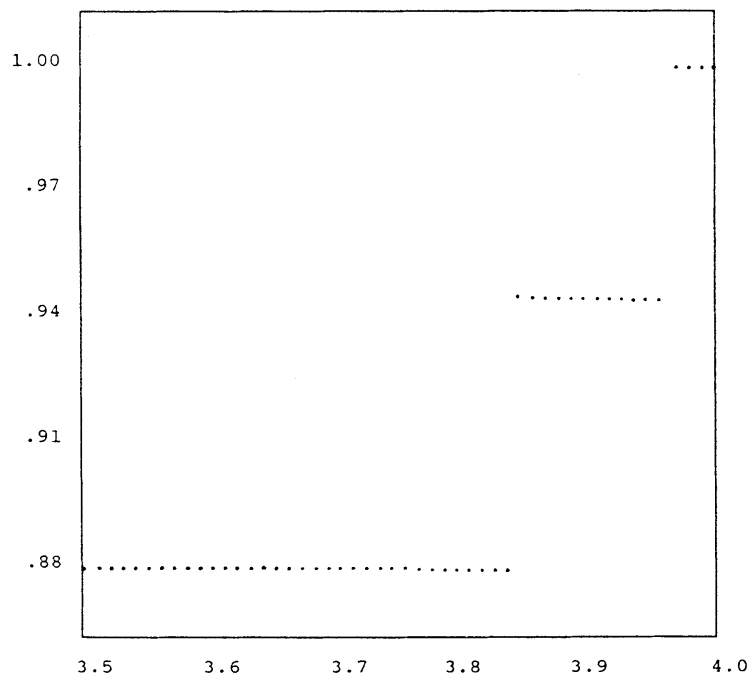

FIGURE 3

Computed topological entropy for the family of discontinuous maps of Example 3

Acknowledgments. The authors are grateful to L. Block for a helpful discussion and to M. Misiurewicz for his remarks and for pointing out to us that Theorem 2 and Corollary 1 are special cases of more general results proved in [15]. 


\section{REFERENCES}

1. P. Collet, J. P. Crutchfield, and J. P. Eckmann, Computing the topological entropy of maps, Comm. Math. Phys. 88 (1983), 257-262.

2. L. Block, J. Keesling, S. Li, and K. Peterson, An improved algorithm for computing topological entropy, preprint 1988.

3. M. Misiurewicz and W. Szlenk, Entropy of piecewise monotone mappings, Studia Math. 67 (1980), 45-63.

4. J. Milnor and W. Thurston, On iterated maps of the interval. I, II, Preprint, Inst. Adv. Studies, Princeton University.

5. R. L. Adler, A. C. Konheim and M. H. McAndrew, Topological entropy, Trans. Amer. Math Soc. 114 (1965), 309-319.

6. L. Block, J. Guckenheimer, M. Misuirewicz and L. S. Young, Periodic orbits and topological entropy of one-dimensional maps, Global Theory of Dynamical Systems, Lecture Notes in Math., vol. 819, Springer-Verlag, New York, 1980, pp. 18-34.

7. T. N. T Goodman, Relating topological entropy and measure-theoretic entropy, Bull. London Math Soc. 3 (1971), 176-180.

8. A. Boyarsky, A matrix method for estimating the Liapunov exponent of one dimensional systems, J. Statist. Phys. 50 (1988), 213-229.

9. P. Góra, A. Boyarsky and H. Proppe, Constructive approximations to densities invariant under nonexpanding transformations, J. Statist. Phys. 51 (1988), 179-194.

10. H. Proppe, W. Byers and A. Boyarsky, Singularity of topological conjugacies between certain unimodal maps of the interval, Israel J. Math. 44 (1983), 277-288.

11. P. Góra and A. Boyarsky, Why computers like Lebesgue measure, Comput. Math. Appl. 16 (1988), 321-329.

12. L. S. Young, Entropy, Lyapunov exponents and Hausdorff dimension in differentiable systems, IEEE Trans. Circuits and Systems CAS-30 (1983), 599-607.

13. L. Block and E. Coven, Approximating entropy of maps of the interval, Dynamical Systems and Ergodic Theory, Banach Center Publications, Polish Sci. Publishers, Warsaw, 1989, pp. 237-242.

14. P. Walters, An introduction to ergodic theory, Springer-Verlag, New York, Heidelberg and Berlin, 1982.

15. M. Misiurewicz, Jumps of entropy in one dimension, Fund. Math. 132 (1989), 215-226.

Department of Mathematics, Warsaw University, Warsaw, Poland

Department of Mathematics, Concordia University, 7141 Sherbrooke Street W, MonTREAL, CANADA H4B 1R6 